\title{
Elevated level of acetylation of APE1 in tumor cells modulates DNA damage repair
}

\author{
Shiladitya Sengupta ${ }^{1,4}$, Anil K. Mantha1,5, Heyu Song ${ }^{2}$, Shrabasti Roychoudhury ${ }^{2}$, \\ Somsubhra Nath ${ }^{2,6}$, Sutapa Ray ${ }^{3}$, Kishor K. Bhakat ${ }^{1,2}$ \\ ${ }^{1}$ Department of Biochemistry \& Molecular Biology, University of Texas Medical Branch, Galveston, TX 77555, USA \\ ${ }^{2}$ Department of Genetics, Cell Biology and Anatomy, University of Nebraska Medical Center, Omaha, NE 68198, USA \\ ${ }^{3}$ Department of Pediatrics, Hematology/Oncology Division, University of Nebraska Medical Center, Omaha, NE 68198, \\ USA \\ ${ }^{4}$ Department of Radiation Oncology, Houston Methodist Research Institute, Houston, TX 77030, USA \\ ${ }^{5}$ Center for Animal Sciences, School of Basic and Applied Sciences, Central University of Punjab, Bathinda 151001, Punjab, \\ India \\ ${ }^{6}$ Molecular Biology Research \& Diagnostic Laboratory, Saroj Gupta Cancer Centre \& Research Institute, Kolkata 700063, \\ India
}

Correspondence to: Kishor K. Bhakat, email: kishor.bhakat@unmc.edu

Keywords: apurinic/apyrimidinic endonuclease 1 (APE1), BER, acetylation, DNA damage repair

Received: April 13, $2016 \quad$ Accepted: September 02, $2016 \quad$ Published: September 19, 2016

\section{ABSTRACT}

Apurinic/apyrimidinic (AP) sites are frequently generated in the genome by spontaneous depurination/depyrimidination or after removal of oxidized/modified bases by DNA glycosylases during the base excision repair (BER) pathway. Unrepaired AP sites are mutagenic and block DNA replication and transcription. The primary enzyme to repair AP sites in mammalian cells is AP endonuclease (APE1), which plays a key role in this repair pathway. Although overexpression of APE1 in diverse cancer types and its association with chemotherapeutic resistance are well documented, alteration of posttranslational modification of APE1 and modulation of its functions during tumorigenesis are largely unknown. Here, we show that both classical histone deacetylase HDAC1 and NAD ${ }^{+}$-dependent deacetylase SIRT1 regulate acetylation level of APE1 and acetylation of APE1 enhances its AP-endonuclease activity both in vitro and in cells. Modulation of APE1 acetylation level in cells alters AP site repair capacity of the cell extracts in vitro. Primary tumor tissues of diverse cancer types have higher level of acetylated APE1 (ACAPE1) compared to adjacent non-tumor tissue and exhibit enhanced AP site repair capacity. Importantly, in the absence of APE1 acetylation, cells accumulate AP sites in the genome and show increased sensitivity to DNA damaging agents. Together, our study demonstrates that elevation of acetylation level of APE1 in tumor could be a novel mechanism by which cells handle the elevated levels of DNA damages in response to genotoxic stress and maintain sustained proliferation.

\section{INTRODUCTION}

Mammalian apurinic/apyrimidinic (AP) endo nuclease 1 (APE1) is a ubiquitous and multifunctional protein. It plays a central role in the repair of AP sites generated either spontaneously or as an intermediate during the repair process of oxidative and drug-induced alkylation damage in the genome via the base excision repair (BER) pathway [1-3]. APE1 cleaves the AP-site to generate $3^{\prime}-\mathrm{OH}$ group and $5^{\prime}$-deoxyribose-5-phosphate
(dRP) termini or removes a 3' blocking group by functioning as a $3^{\prime}$ exonuclease [4]. In addition to its DNA repair function, APE1 functions as a transcriptional coregulator of many genes involved in multiple cellular pathways [5-11]. APE1 was also shown to activate DNA-binding of many stress-inducible transcription factors (including c-Jun, P53 and NF-kB) through its redox effector (Ref-1) function [11-13]. Thus, APE1 has a critical role in linking DNA repair to regulation of diverse signaling pathways. 
We discovered earlier that human APE1 is acetylated primarily at Lys 6 and Lys 7 by the histone acetyltransferase p300 both in vitro and in cells [14]. Acetylation modulates the transcriptional regulatory function of APE1 in up- and downregulation of diverse genes associated with multidrug resistance, cell-cycle control and apoptosis [5, 9, 15]. Dr. Tell's group in collaboration with us have shown that other Lys residues (Lys 27, 31, 32, 35) in the N-terminal domain of APE1 can also be acetylated upon genotoxic stress and mutation of these Lys residues to Ala alters the DNA damage repair activity of APE1 [16]. APE1 was also found to be ubiquitinated at multiple Lys (Lys 24, 25, 27) residues in the $\mathrm{N}$-terminal domain and ubiquitination at these residues can modulate the stability or localization of APE1 $[17,18]$. Other posttranslational modifications such as phosphorylation and nitrosylation have been shown to alter multiple functions of APE1 [18-22]. The disordered and conserved N-terminal domain of APE1 harboring the multiple acetylation sites is the common interaction domain for multiple partners in diverse pathways including transcriptional regulation [5, 7-10], and RNA processing $[23,24]$. Importantly, we discovered that both the DNA repair function and acetyl-acceptor Lys 6 and 7 sites in APE1 are essential for cell proliferation and survival [25]. Similarly, other BER proteins, including NEIL2 and OGG1 have also been found to be acetylated, modulating their DNA repair function $[26,27]$.

Overexpression of APE1 in cancer cell lines and tumour tissues from various sources including non-small cell lung cancer (NSCLC), colon, glioma, head and neck, breast, and its association with resistance to various anticancer drugs strongly establishes APE1 as a target for cancer therapy [28-36]. However, little is known about alteration of posttranslational modifications of APE1 during tumorigenesis. Recently, we have shown that the N-terminal domain (1-33 amino acids; aa) of APE1 is cleaved by a limited proteolysis in tumor, acetylation of multiple Lys residues in this domain prevents this proteolysis [37].

Here, we examined the regulation of acetylation of APE1 in cells by the interplay of both classical and NAD ${ }^{+}$ dependent histone deacetylases. We found that acetylation increases the DNA repair activity of APE1, and absence of this acetylation contributes to accumulation of AP sites in the genome and increased cell sensitivity towards both alkylating and oxidative agents. Primary tumor tissues of various cancer types have elevated levels of AcAPE1 and exhibit significantly enhanced AP site repair capacity. Together, our study suggest that increased levels of AcAPE1 in tumor plays a critical role in their survival and sustained proliferation in response to genotoxic stress.

\section{RESULTS}

\section{Elevated levels of AcAPE1 in tumor tissue}

We compared AcAPE1 level in primary tumor tissues to adjacent non-tumor (normal) tissues from patients with colon, non-small cell lung cancer (NSCLC) or pancreatic cancer by Western blot analysis (Figure 1A, 1B \& 1C) using our previously generated AcAPE1-specific antibody [5]. We have previously shown that this antibody is highly specific in recognizing AcAPE1 species (acetylated at Lys 6 position) and does not cross react with 50 -fold excess of unmodified APE1 [5]. We found that the fraction of APE1 present in acetylated form (AcAPE1/ total APE1) was significantly higher in tumor tissues as compared to adjacent non-tumor tissues (Figure 1D and Supplementary Figure S1A, S1B \& S1C). Immunohistochemical analysis also confirmed increased nuclear AcAPE1 staining in tumor compared to non-tumor tissues (Figure 1E). These data indicate that tumor tissues of diverse cancer types have elevated levels of AcAPE1 as compared to the adjacent non-tumor tissues.

\section{Both classical histone deacetylase HDAC1 and $\mathrm{NAD}^{+}$-dependent deacetylase SIRT1 are involved in deacetylation of APE1 in cells}

Deregulation of the fine balance between histone acetyl transferases and deacetylases in tumor cells may affect the acetylation level of APE1 during tumorigenesis. We showed previously that p300 is the major acetyltransferase for acetylation of APE1 at Lys 6 and 7 residues in cells [14]. To ask whether classical histone deacetylases (HDAC1-11) are also involved in deacetylation of AcAPE1, we treated colon adenocarcinoma HCT116 cells with trichostatin A (TSA), a specific inhibitor for classical HDACs [38], and measured AcAPE1 level. We found that TSA treatment significantly increased the level of AcAPE1 ( $>$ 5-fold) after as little as 1 hour (h) of treatment (Figure 2A, upper panel; Supplementary Figure S2A), without changing the total level of APE1 (Figure 2A, lower panel). AcAPE1 returned to the normal level by $24 \mathrm{~h}$. Similarly, TSA treatment increased AcAPE1 levels in hTERT-transformed human primary skin fibroblast BJ cells (Figure 2B; Supplementary Figure S2B). These data indicate that classical deacetylases are involved in regulating acetylation of APE1 in cells. Because NAD ${ }^{+}$dependent non-classical histone deacetylase SIRT1 was also shown to be involved in deacetylation of AcAPE1 [39], we found that treatment with $\mathrm{NAD}^{+}$-dependent deacetylase inhibitor nicotinamide (NAM) increased the AcAPE1 level, while combined treatment of both NAM and classical deacetylase inhibitor TSA had an additive effect (Figure 2C; Supplementary Figure S2C). Thus both classical and $\mathrm{NAD}^{+}$-dependent HDACs along with p300 [14] are involved in maintaining the homeostasis of the acetylation/deacetylation cycles of APE1 in cells.

Our earlier studies showed that APE1 is associated with Class I (HDAC1-3) but not with Class II (HDAC 4-6) deacetylases [14]. We asked which HDAC deacetylates AcAPE1 in vitro. At first, we generated AcAPE1 in vitro 
by incubating purified recombinant wild type (WT) APE1 [5] with p300 HAT domain either in the presence or absence of acetyl coenzyme A (AcCoA), and confirmed acetylation by Western blot analysis using our AcAPE1 specific antibody (Figure 3A). Next, we incubated in vitro AcAPE1 with affinity-purified FLAG-HDACs (from HCT116 cells ectopically expressing these individual HDACs), followed by examination of AcAPE1 level by Western blot analysis. We found that only HDAC1 but not HDAC2-6 can efficiently catalyze deacetylation of AcAPE1 in vitro (Figure 3B; Supplementary
Figure S3A). To further confirm that HDAC1 is involved in regulating the acetylation level of APE1 in cells, we downregulated endogenous HDAC1 level using HDAC1-specific siRNA. Figure 3C and Supplementary Figure S3B show that downregulation of HDAC1 level significantly increased APE1 acetylation. Consistent with this, ectopic expression of HDAC1 reduced AcAPE1 level (Figure 3D \& 3E; Supplementary Figure S3C, S3D \& S3E), and combined ectopic expression of HDAC1 and SIRT1 reduced AcAPE1 level to a greater extent (Figure 3E; Supplementary Figure S3E). Moreover,

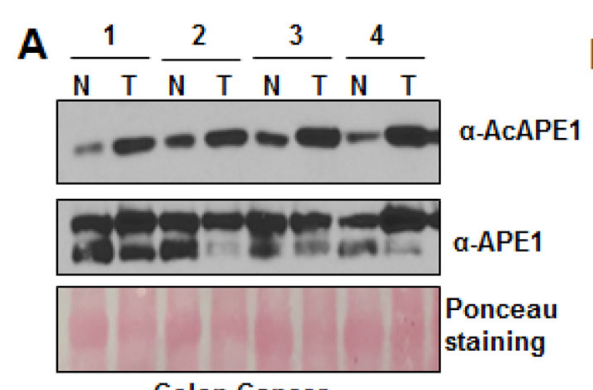

Colon Cancer

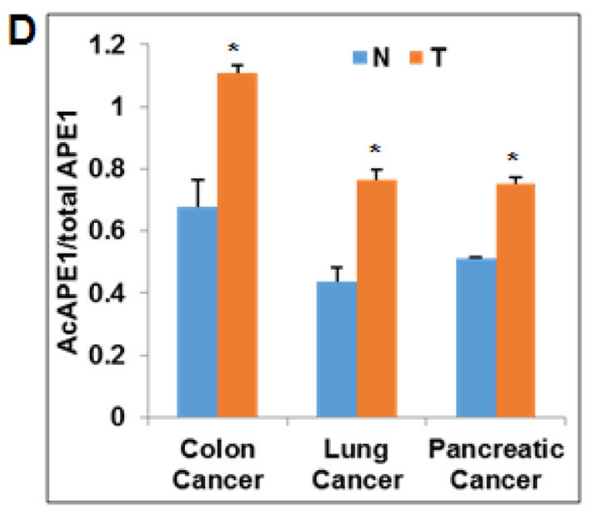

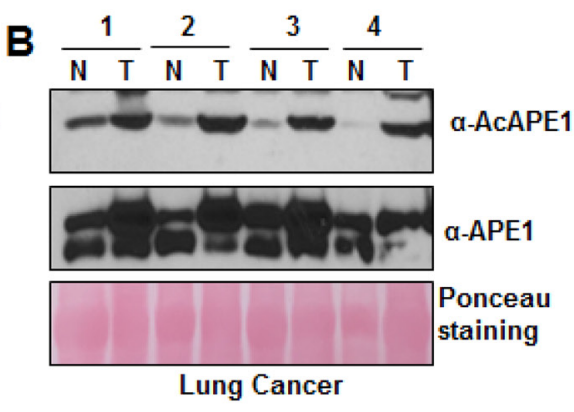

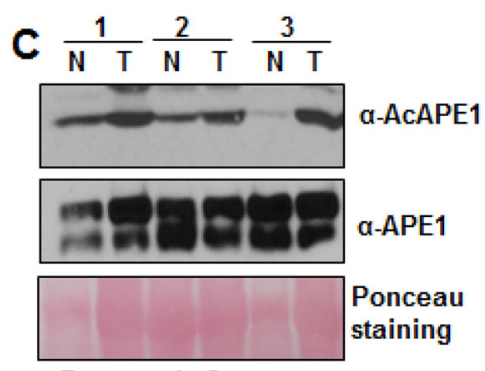

Pancreatic Cancer
E

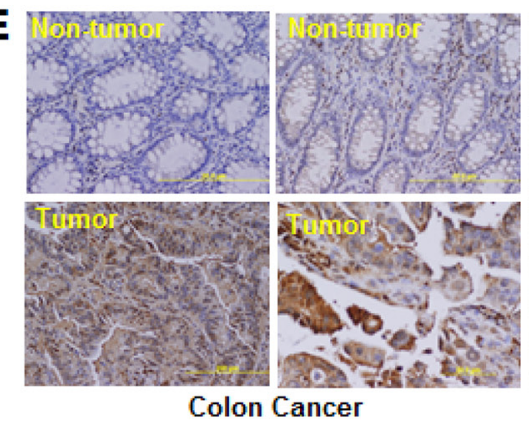

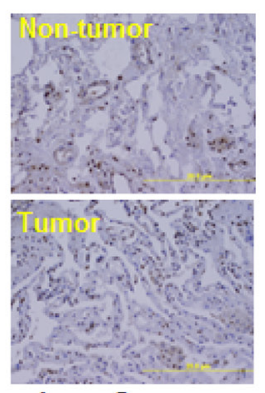

Lung Cancer

Figure 1: Elevated levels of AcAPE1 in tumor tissue. A, B \& C. Western blot analysis of tumor-adjacent non-tumor (N) and tumor (T) tissue lysates from colon (A), lung (B) and pancreatic (C) cancer patients with $\alpha$-AcAPE1, APE1 Abs; ponceau staining used as loading control. D. The levels of APE1 and AcAPE1 in these tissue samples were quantitated using ImageJ and the ratio of AcAPE1/total APE1 was represented in a histogram with mean $\pm \mathrm{SD} ; \mathrm{p}$ value $<0.05$ shown as * (Student T Test). E. Representative immunohistochemical staining of AcAPE1 in paraffin-embedded colon and lung cancer tissue sections.
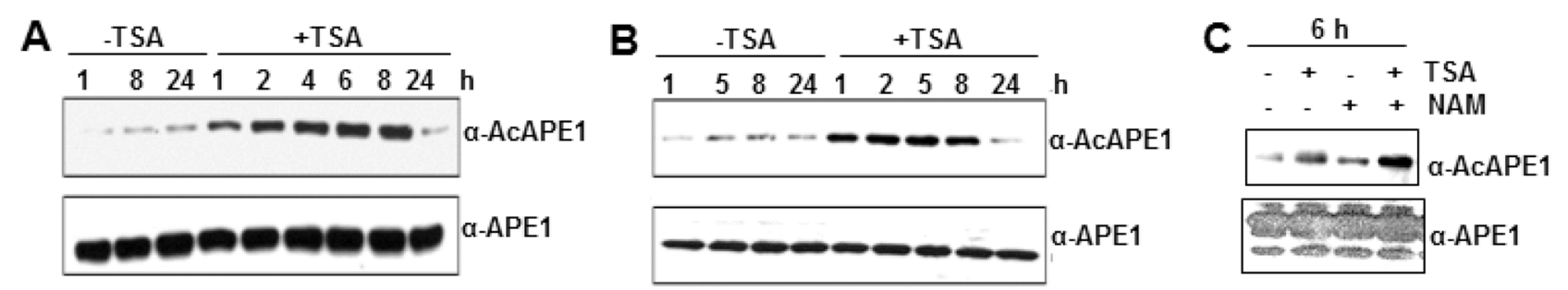

Figure 2: Effect of classical HDAC inhibitor TSA and non-classical deacetylase inhibitor NAM on APE1 acetylation in cells. A \& B. HCT116 (A) and hTERT-BJ (B) cells were treated with TSA (100 ng/ml) and the levels of AcAPE1 (upper panel) or APE1 (lower panel) were measured at the indicated time points by Western blot analysis. C. HCT116 cells were treated with either $100 \mathrm{ng} / \mathrm{ml}$ TSA or $10 \mathrm{mM}$ NAM or both for $6 \mathrm{hrs}$ and the level of AcAPE1 (upper panel) or APE1 (lower panel) were measured by Western blot analysis. 
the effect of ectopic HDAC1 expression on AcAPE1 level was overcome in the presence of deacetylase inhibitor TSA (Figure 3F; Supplementary Figure S3F). Although resveratrol was shown to activate SIRT1 [40], treatment with resveratrol did not decrease AcAPE1 level (Figure 3F). Together, these data indicate that both classical HDAC1 and $\mathrm{NAD}^{+}$-dependent HDAC are involved in maintaining the homeostasis of acetylation/ deacetylation cycles of APE1 in cells.

\section{Modulation of acetylation level of APE1 in cells affects AP site cleavage activity in vitro}

To explore whether modulation of APE1 acetylation level in cells affects its DNA repair activity, we treated HCT116 cells with TSA and NAM for $6 \mathrm{~h}$ and then measured the AP-endonuclease activity in cell extracts using THF containing oligonucleotide substrate as described before [41]. A dose-dependent increase in the THF cleavage activity of cell extracts was observed after treatment with TSA and NAM (Figure 4A). Because treatment with TSA and NAM enhanced AcAPE1 level without changing the total APE1 (Figure 2C), these results indicate that the increased AP site cleavage activity of TSA and NAM treated cell extracts is due to enhanced APE1 acetylation. Furthermore, we ectopically expressed HDAC1 and SIRT1 (which are primarily involved in deacetylation of APE1; Figure 3; [39]), and measured the AP-endonuclease activity of the cell extracts. Ectopic expression of either HDAC1 or SIRT1 decreased the THF cleavage activity of the cell extracts (Figure 4B; Supplementary Figure S4). Thus modulation of acetylation levels of APE1 in cells plays a key regulatory role in AP site repair capacity.
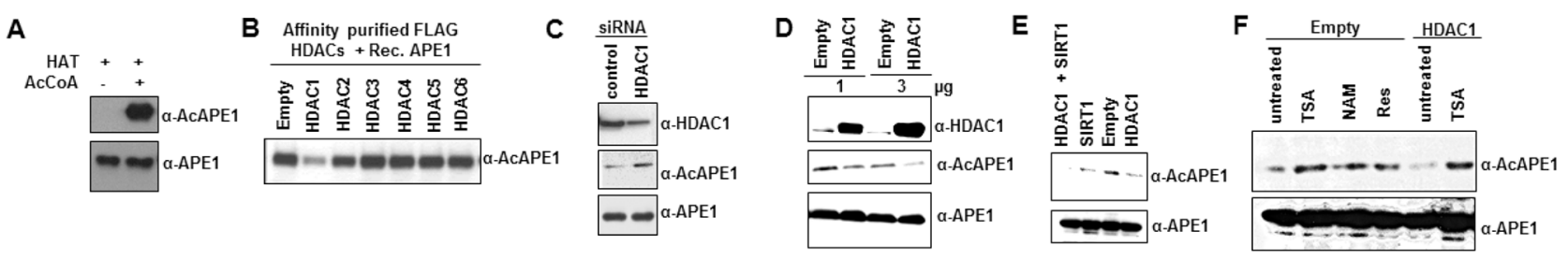

Figure 3: HDAC1 and SIRT1 are responsible for APE1 deacetylation. A. Recombinant APE1 (2 $\mu \mathrm{g})$ was incubated with p300 HAT domain $\pm 1 \mathrm{mM} \mathrm{AcCoA}$ for $2 \mathrm{~h}$ at $30^{\circ} \mathrm{C}$ and then $50 \mathrm{ng}$ of unmodified or acetylated APE1 was used for Western blot analysis with Abs specific for AcAPE1 or APE1. B. Recombinant in vitro acetylated APE1 (200 ng) was incubated with immunoprecipitated FLAGHDACs (eluted with FLAG peptide) from FLAG-HDAC1-6 transfected HEK293T cells. The levels of AcAPE1 were measured by Western blot analysis. C. HEK293T cells were transfected with $80 \mathrm{nM}$ of HDAC1 specific or control duplex siRNA. HDAC1, AcAPE1 and APE1 levels in cell extracts were measured $48 \mathrm{~h}$ after transfection by Western blot analysis. D. HEK293T cells were transfected with 1 or $3 \mu \mathrm{g}$ of HDAC1 expression plasmid. Forty eight hours after transfection the AcAPE1 and APE1 levels were measured in the cell extracts by Western blot analysis. E. HEK293T cells were transfected with either SIRT1 or HDAC1 or both expression plasmids and then the level of AcAPE1 and APE1 were measured by Western blot analysis. F. HEK293T cells were transfected with either empty vector or HDAC1 expression plasmids and then treated with TSA, NAM or resveratrol as indicated and the levels of AcAPE1 and APE1 were measured by Western blot analysis.
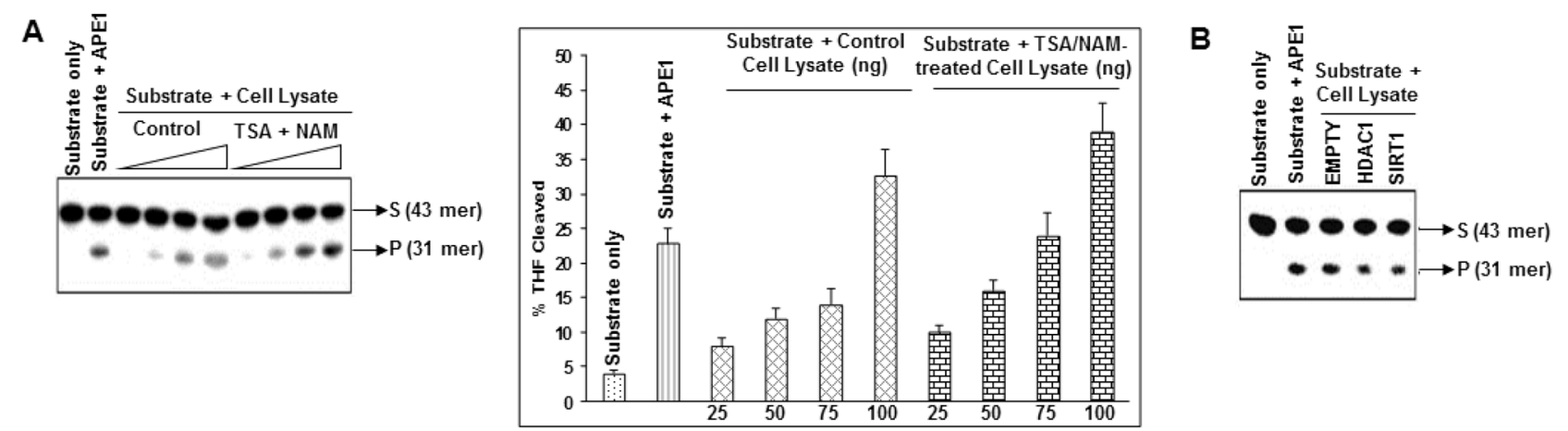

Figure 4: Modulation of AcAPE1 levels in cells alters AP site repair capacity of their extracts. A. HCT116 cell were treated with or without TSA $(100 \mathrm{ng} / \mathrm{ml})$ and NAM $(10 \mathrm{mM})$ for $6 \mathrm{hrs}$. Various amount $(25-100 \mathrm{ng})$ of cell extracts was incubated with $5^{, 32} \mathrm{p}$-labeled THF (reduced AP site) containing 43-mer duplex oligonucleotide (substrate: S) at $37^{\circ} \mathrm{C}$ for 3 min, and the cleaved product (P) was separated as described in Materials \& Methods; side panel: the percentage of cleaved product from the substrate was quantitated and plotted with \pm SD from 2-3 independent experiments. B. HCT116 cells were transfected with empty vector or HDAC1 or SIRT1 expression plasmids and $48 \mathrm{~h}$ after transfection, $100 \mathrm{ng}$ cell extracts from each sample were used for AP-endonuclease activity assay as described in (A). 


\section{Acetylation of APE1 enhances its endonuclease activity}

The observation that modulation of AcAPE1 level in cells alters AP site repair capacity of cell extracts in vitro (Figure 4), raises the possibility that acetylation of APE1 could modulate its DNA damage repair activity. We investigated directly whether acetylation affects the AP-endonuclease activity of APE1 in vitro. We found that acetylation of APE1 increased its AP-endonuclease activity in a dose-dependent manner (Figure 5A). Consistent with this we observed that mutation of two acetylable Lys $6 \& 7$ to acetylation-mimic Gln (K6Q/ $\mathrm{K} 7 \mathrm{Q}$ ) which eliminates the positive charge and resemble acetyl-Lys, significantly increased APE1's endonuclease activity in vitro (Figure 5B; Supplementary Figure S5A). However, to our surprise we observed that mutation of Lys $6 \& 7$ to neutral aa Leu (K6L/K7L) which eliminates the positive charge but does not resemble acetyl-Lys, could not enhance AP-endonuclease activity. These results suggest that acetylation of APE1 enhances its AP endonuclease activity.

\section{Tumor tissue extracts have increased AP-site cleavage activity}

Consistent with our observation that acetylation significantly increased endonuclease activity of APE1 (Figure 5A \& 5B), extracts of NSCLC tissue samples showed significantly enhanced AP site cleavage activity with respect to matched non-tumor control when tissue extracts with equivalent amount of APE1 were used in in vitro cleavage assay (Figure 5C \& 5D; Supplementary Figure S5B). This suggests that the observed higher AcAPE1 level in tumor tissues contributes to enhanced AP site repair capacity of their extracts.

\section{Absence of acetylation of APE1 contributes to accumulation of AP sites in the genome}

The observation that acetylation of APE1 enhances its endonuclease activity raises the possibility that in the absence of acetylable Lys residues in APE1, cells will accumulate AP sites in the genome. We quantitated AP sites in the genome of cells expressing WT and
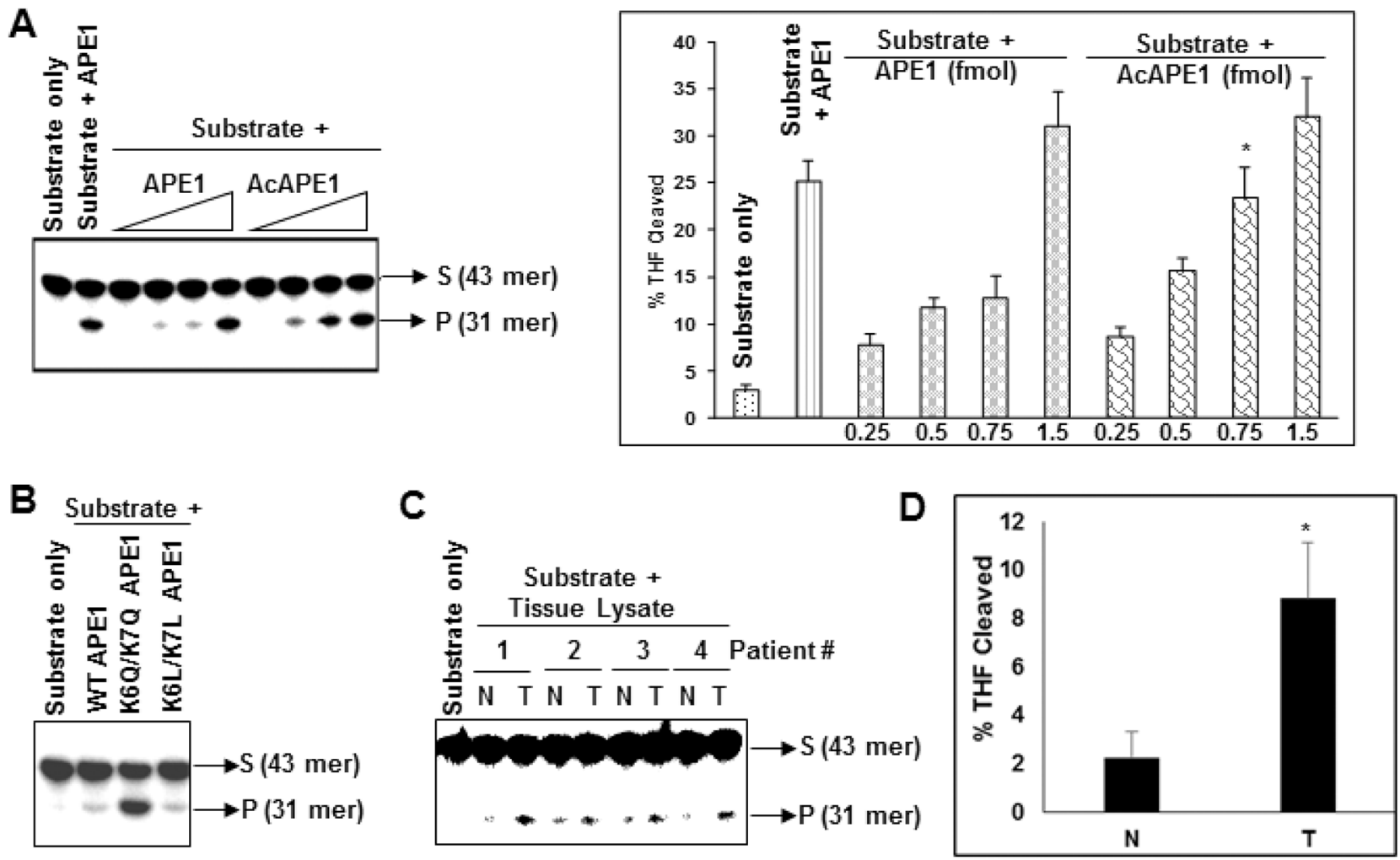

Figure 5: Acetylation of APE1 enhances its AP-endonuclease activity and tumor tissues have higher AP-endonuclease activity. A. Incision of the ${ }^{3}{ }^{32} \mathrm{p}$-labeled THF (reduced AP site)-containing 43-mer duplex oligonucleotide by $0.1,0.5,1.0$ or 5.0 fmol of recombinant APE1 or AcAPE1; side panel: quantitation of the percentage of cleaved product from the substrate shown. B. Comparison of AP-endonuclease activity of WT, acetylation mimic K6Q/K7Q (QQ) or K6L/K7L (LL) mutant APE1 proteins. C. Tissues extracts containing equal amount of APE1 from non-tumor $(\mathrm{N})$ or tumor $(\mathrm{T})$ tissues of lung cancer patients were incubated with $5^{, 32} \mathrm{p}$-labeled THF-containing duplex oligo and the cleaved product was separated as described before. D. Quantitation of the percentage of THF cleaved product in the non-tumor and tumor samples from $\mathrm{C}$ and plotted with $\pm \mathrm{SD}$. 
non-acetylable K5R (acetylable Lys 6,7,27,31,32 mutated to Arg) mutant by using an aldehyde-reacting probe as described elsewhere [42-44]. We used HEK293T ${ }^{\text {APE1siRnA }}$ cells stably expressing APE1 siRNA under doxycycline (Dox)-inducible promoter [9]. We treated the cells with Dox to downregulate endogenous APE1 and then ectopically expressed FLAG-tagged WT APE1, or its mutant lacking the acetylation sites [37]. We observed that depleting endogenous APE1 increased AP sites in the genome compared to control (Figure 6A). Importantly, a significant increase in AP sites was observed in the genome of cells expressing K5R APE1 mutant compared to WT APE1 expressing cells (Figure 6A). Furthermore, we treated these cells with glucose oxidase (GOx) which produces hydrogen peroxide and induces oxidative damages which in turn produces AP sites in the genome $[27,45,46]$. We first confirmed that GOx treatment indeed induced oxidative DNA damage by Fpg-alkaline Comet assay. As shown in Figure 6B, treatment with GOx enhanced significant DNA breaks in the genome as evidence by increased comet tail in GOx-treated cells as compared to control. Consistent with this, treatment with GOx significantly enhanced AP sites in the genome of APE1 downregulated cells which can be relieved by ectopic expression of WT APE1 but not by the nonacetylable K5R mutant (Figure 6C). This indicates that APE1 acetylation plays an important role in DNA damage or AP site repair in cells. Furthermore, we also quantitated AP sites in control and APE1-downregulated HCT116 cells that stably express APE1-specific shRNA. As expected, we found that downregulation of endogenous APE1 significantly accumulated AP sites in the genome which was rescued by ectopic expression of WT APE1 but not by the K5R mutant APE1 (Figure 6D).

\section{APE1 acetylation plays a role in cell survival in response to genotoxic stress}

We examined the role of APE1 acetylation in cell survival and/or proliferation. As expected, we observed that depleting endogenous APE1 with Dox significantly decreased the number of viable HEK293T ${ }^{\text {APE1 siRNA }}$ colonies compared to control (Figure 6E). This effect can be rescued by ectopic expression of WT APE1, but not with non-acetylable K5R mutant APE1 (Figure 6E). We further examined the role of APE1 acetylation in cell proliferation after induction of DNA damage. Treatment with GOx decreased the number of viable colonies which can be
A

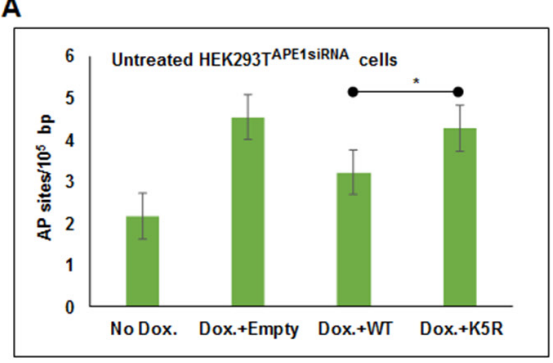

D

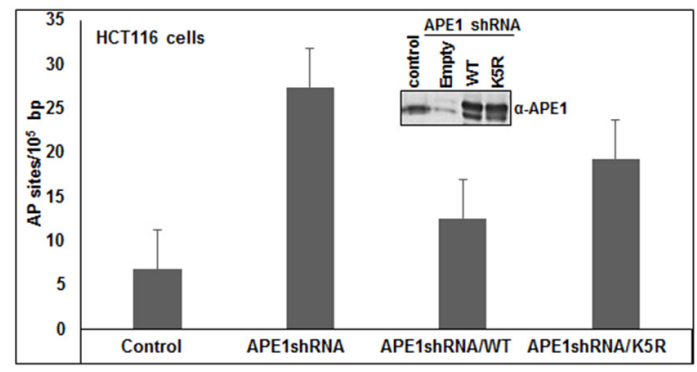

B

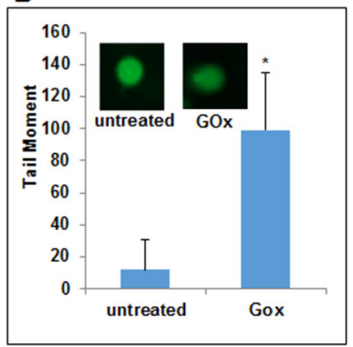

E
C

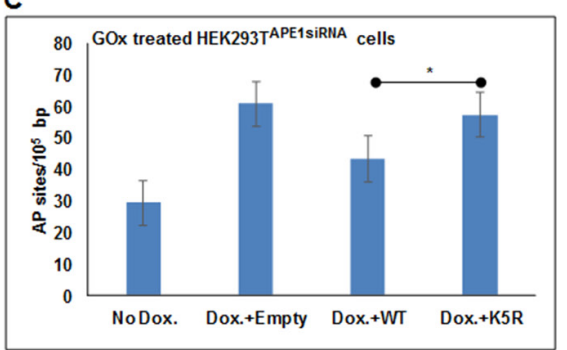

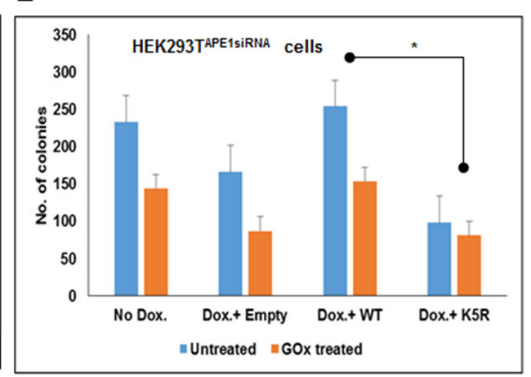

$\mathbf{F}$

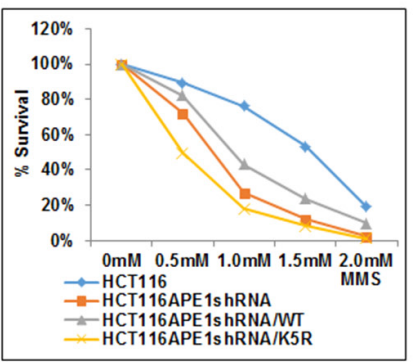

Figure 6: Absence of APE1 acetylation leads to accumulation of AP sites in the genome and sensitizes cells to DNA damaging agents. A \& C. Endogenous APE1 was downregulated in HEK293T ${ }^{\text {APElsiRnA }}$ cells with Dox treatment. FLAG-tagged WT or acetylation defective (mutations of Lys 6, 7, 27, 31 \& 32 to non-acetylable Arg; K5R) mutant APE1 was ectopically expressed in these cells. AP sites in the genomic DNA were measured using ARP kit in glucose oxidase (GOx; $100 \mathrm{ng} / \mathrm{ml}$ for $30 \mathrm{mins}$ ) treated (C) or untreated control (A) cells. Bar diagram representing number of AP site $/ 10^{5} \mathrm{bp}$. Error bars indicate mean $\pm \mathrm{SD}(\mathrm{n}=3)$. B. FPG Comet assay to assess relative quantitation of oxidative DNA damage after GOx treatment. Histogram showing mean tail moment (50-100 randomly selected cells) after GOx treatment; representative images of Comet slides shown in inset. D. Quantitation of AP sites in control, APE1 shRNA expressing HCT116 cells, WT or K5R mutant APE1 expressing HCT116 cells after endogenous APE1 downregulation (expression level shown in inset) as in A \& C. E \& F. DNA damage cell sensitivity assay measured by clonogenic assay (E) after GOx treatment in endogenous APE1 downregulated HEK293 $\mathrm{T}^{\mathrm{APE} 1 \text { siRNA }}$ cells (by Dox treatment) and after ectopic expression of WT or K5R mutant APE1in endogenous APE1 downregulated cells, and (F) in APE1 shRNA expressing HCT116 cells transfected with WT or K5R APE1 and treated with different dose of MMS and plotted as \% survival. Details are in Materials and Methods. * represents $\mathrm{p}$ value $<0.05$ (Student T Test). 
rescued by ectopic expression of WT APE1, but not with its non-acetylable mutant (Figure 6E). Furthermore, we found that treatment with alkylating agent methylmethane sulphonate (MMS) sensitized HCT116 cells that express APE1-specific shRNA (Figure 6F). While ectopic expression of WT APE1 protected these cells to some extent, expression of non-acetylable K5R mutant sensitized the cells to MMS (Figure 6F). These data indicate that APE1 acetylation plays a critical role in cell survival and/or proliferation and that the absence of APE1 acetylation sensitizes cells to DNA damaging agents.

\section{DISCUSSION}

Despite the fact that the N-terminal acetylable Lys residues in APE1 are dispensable for its DNA repair activity in vitro [47], this study unraveled the novel role of acetylation of APE1 in AP site repair both in vitro and in cells. Furthermore, our study provides evidence that alteration of acetylation level of APE1 in diverse primary tumor tissues plays a critical role for cell survival and proliferation. AP sites in the genome are generated spontaneously or after excision of the damaged bases by DNA glycosylases during BER [2, 3, 48]. Approximately, 10,000 such lesions are continuously generated endogenously in the genome/cell/day [49]. Unrepaired AP sites block DNA replication and transcription and are highly susceptible to spontaneous cleavage and thus DNA strand breaks, leading to cell apoptosis [50-54]. Thus the DNA repair function of APE1 plays an essential role to protect cells from both endogenous and exogenous DNA damage. Our current study shows that acetylation enhances its AP-endonuclease activity and plays an essential role in cell proliferation and survival in response to DNA damages.

Tumor cells are proliferating, transcriptionally very active and have energy demands, which produce oxidative stress $[55,56]$. All these factors contribute to enhanced generation of AP sites in the genome of tumor cell [57-60]. Presence of oxidative damage and AP site clusters in replicating or transcriptionally active unfolded chromatin regions may be prevalent in tumor. Thus, there is urgency to repair AP sites faster and efficiently in tumor cells for their survival and proliferation. The AP-endonuclease activity of APE1 should be enhanced in order to repair increased AP sites in tumor cells, which can be achieved by enhancing its acetylation. Consistent with this idea, our data show that primary tumor tissue of diverse origins has higher acetylated APE1 compared to non-tumor tissue. Thus, our study suggests that alteration of posttranslational modification (i.e. acetylation) of APE1 could be a novel mechanism that cancer cells exploit to handle the elevated levels of DNA damage and maintain sustained proliferation. Enhanced APE1 acetylation after treatment with genotoxic agents such as MMS or GOx $[5,9,16,39]$ supports this scenario. Moreover, given our and other's findings that APE1 acetylation modulates expression of many genes [5, 6, 9, 14, 37], and it enhances its endonuclease activity (present study), it appears that increased APE1 acetylation in tumor cells modulates DNA damage repair, and at the same time also alters gene expression to maintain sustained proliferation.

Although earlier studies showed that the N-terminal domain (1-63 aa) of APE1 is not essential for its APendonuclease activity in vitro [47], one recent study suggests that neutralization of many charged Lys residues in this domain enhances its DNA repair activity [16]. However, whether posttranslational modification of these Lys residues modulates this function is largely unknown. In this study we show directly that covalent modification of these Lys residues by acetylation modulates DNA repair activity of APE1 both in vitro and in cells. Stimulation of APE1's AP-endonuclease activity due to acetylation suggests two possible mechanisms: acetylation in APE1 could either increase its affinity for the substrate AP site in DNA, or decrease APE1's affinity for the product (cleaved AP-site), thus increasing its turnover. Because APE1 remains bound to its product, and that the ratelimiting step in AP site excision is dissociation of APE1 from the product [61-63], it is likely that acetylation enhances APE1's turnover by weakening its interaction with the AP site product. Because acetylation is one of the posttranslational modifications by which the overall positive charge of a protein is neutralized, and Lys 6, 7, 27, 31 and 32 in APE1 can be modified by acetylation, we propose that acetylation of APE1 likely decreases highly positive "charge patch" in its N-terminal domain. This in turn weakens or abrogates the charge-dependent contacts of the N-terminal domain of APE1 with the product cleaved AP site. Interestingly, mutation of the acetylable Lys 6 \& 7 to neutral Leu, which eliminates the positive charge but does not resemble acetyl Lys have no significant effect on its AP-endonuclease activity. This also raises the possibility that acetylation not only neutralizes the positive charges in the N-terminal but also puts acetyl marks which may induce conformational changes in APE1 that facilitates substrate binding or release from the product cleaved AP sites. Further studies are necessary to address this issue.

Posttranslational modifications have emerged as the main mechanisms for controlling multiple functions of a protein. Like acetylation, other posttranslational modifications such as phosphorylation, ubiquitination and nitrosylation of APE1 were reported earlier. An earlier study by Yacoub et al. [64] showed that APE1 can be phosphorylated in vitro by casein kinase I and II (CKI and CKII). CKII-mediated phosphorylation of APE1 abolished DNA repair activity in vitro, while phosphorylation by PKC was shown to alter the redox function of APE1 [64, 65]. A subsequent study by Fritz and Kaina showed that APE1 phosphorylation by CKII enhances redox activation of the AP-1 transcription 
factor and has no effect on its DNA repair activity [66]. Although these studies reported APE1 phosphorylation by CK II in vitro and PKC in cells, the phosphorylation sites have not been identified. However, one study has shown that APE1 can also be phosphorylated in human cells at $\mathrm{Thr} 233$ in the C-terminal domain by Cdk5 and that phosphorylation reduced the AP-endonuclease activity both in vitro and in cells [22]. However, we found that mutation of Thr 233 to phosphomimic Glu had no effect on APE1's acetylation compared to the WT protein (data not shown). Ubiquitination of APE1 at multiple Lys (Lys $24,25,27)$ residues in the $\mathrm{N}$-terminal domain was shown to modulate the stability or localization of APE1 [17-19]. Because acetylation and ubiquitination occurs in the same Lys residues in many proteins and regulate their stability, we examined whether acetylation of APE1 affects its halflife. Surprisingly, we found that different doses of protein translation inhibitor cycloheximide had not much effect on endogenous APE1 as well as ectopic APE1 protein level (Supplementary Figure S6). Moreover, we found that, mutation of the acetylable Lys residue to Arg in APE1 had no significant effect on the stability of APE1 (Supplementary Figure S6C \& S6D). We do not know the reason why treatment of cycloheximide showed no effect on APE1 level. Other method such as metabolic labeling of APE1 with $\mathrm{S}^{35}$ methionine may enable to resolve this issue. $S$-nitrosylation of APE1, another post-translational modification, has been shown to occur in vivo. Treatment with $S$-nitroglutathione, an $S$-nitrosylating agent, stimulated nuclear export of APE1 through $S$-nitrosylation at Cys93 and Cys310 in a CRM1-independent manner [20]. Thus it is evident that the activity of APE1 or its localization could be finely tuned via different posttranslational modifications, including phosphorylation, acetylation, nitrosylation, ubiquitination, etc., in order to coordinate specific biological functions.

Over the last 20 years, overexpression of APE1 and alteration of its subcellular localization in many cancer types have been established. However, our study provides strong evidence that primary tumor of various cancer types have elevated levels of AcAPE1. The mechanism behind increase in APE1 acetylation level in tumor is currently not known. Because p300 primarily acetylates APE1 in cells [14], and both classical histone deacetylase HDAC1 and $\mathrm{NAD}^{+}$-dependent deacetylase SIRT1 are involved in APE1 deacetylation in cells $[14,39]$, deregulation of the fine balance between acetyl transferase p300 and HDAC1/SIRT1 deacetylase activity in tumor may elevate APE1 acetylation during tumorigenesis. However, our results on p300, HDAC1 and SIRT levels in non-tumor vs. tumor tissues (data not shown) could not explain enhanced APE1 acetylation in tumor cells. We have recently shown that acetylation level of APE1 increases in S-phase of the cell cycle [37], when p300 is also activated [67], and thus cell proliferation could be a trigger for enhanced APE1 acetylation in cancer Furthermore, enhanced phosphorylation of APE 1 by PKC or Cdk 5 which is often overexpressed in some cancers may alter the acetylation level of APE1 [22, 68]. Several studies have shown that multiple signal-dependent modifications (phosphorylation, acetylation, etc.) of a protein occur sequentially, with one modification influencing the subsequent ones [69]. Because APE1 is known to be phosphorylated [65], it is possible that such modification of APE1 in cancer cells enhances its interaction with p300 to induce acetylation or decrease its interaction with HDAC1/SIRT1 to maintain enhanced level of APE1 acetylation.

In summary, this study shows that APE1 acetylation enhances its AP-endonuclease activity and that enhanced acetylation of APE1 in tumors could contribute in protecting cells from endogenous and drug-induced DNA damages. Because APE1 acetylation can modulate both its transcriptional regulatory functions and DNA damage repair function, our study implicates that the AcAPE1 rather than total APE1 levels in tumor could be used as a predictive marker in cancer. Although extensive studies are being carried out on targeting the DNA repair or redox function of APE1 to sensitize tumor cells [70-74], our study provides a rationale for future development of AcAPE1 targeted therapeutic regimens.

\section{MATERIALS AND METHODS}

\section{Patients' tissue samples, extraction of tissue lysates and western blot analysis}

The resected frozen tissues from non-small cell lung carcinoma (NSCLC), colon and pancreatic cancer patients (both tumor and adjacent non-tumor) were collected from University of Texas Medical Branch (UTMB; Galveston) Cancer Center tissue bank. Tissues were collected in accordance with institution's review board approval. Cell lysates were prepared with approximately $100 \mathrm{mg}$ of tissue, washed in phosphate buffered saline (PBS) pH 7.4. Each tissue was minced into fine pieces and homogenized using a glass dounce homogenizer in $1 \mathrm{ml}$ of cold lysis buffer containing $50 \mathrm{mM}$ Tris- $\mathrm{HCl} \mathrm{pH} 7.5,150 \mathrm{mM} \mathrm{NaCl}$, $1 \%$ Triton X-100, $0.1 \mathrm{mM}$ EDTA and protease inhibitor cocktail buffer tablet (PI; Roche Diagnostics). Lysates were centrifuged at $14,000 \mathrm{rpm}$ for $20 \mathrm{~min}$ at $4^{\circ} \mathrm{C}$ and the supernatants were stored at $-80^{\circ} \mathrm{C}$. Tissue extracts equivalent to 30-60 $\mu \mathrm{g}$ of total protein were separated by SDS/PAGE, transferred to nitrocellulose membrane for Western blot analysis with 1:2000 dilution of mouse monoclonal $\mathrm{Ab}$ (Novus Biologicals; \# NB100-116) and 1:300 dilution of rabbit polyclonal acetylated APE1 (AcAPE1) Ab [5]. Bands were quantitated using Image $J$ analysis tool.

\section{Cell lines, plasmids, siRNAs, transfection and treatments}

Human embryonic kidney HEK293T (ATCC \# CRL3216) and doxycycline inducible APE1 downregulated 
APE1siRNAHEK293T cells were cultured in DMEM-high glucose medium with 10\% fetal calf serum (FCS; Sigma) and antibiotic mixture of $100 \mathrm{U} / \mathrm{ml}$ penicillin and 100 $\mu \mathrm{g} / \mathrm{ml}$ streptomycin (Gibco-BRL) as described previously [9]. Human colon cancer HCT116 (ATCC \#CCL-247) cells were grown in MCoy's 5A medium. Generation of HCT116 cells stably expressing APE1 shRNA or control shRNA were described previously [15]. hTERT-immortalized human foreskin fibroblast BJ-5ta (ATCC \#CR-4001) cells were cultured in DMEM-low glucose medium (Gibco-BRL) with FCS and antibiotics. All cell lines were authenticated by STR DNA profiling on August, 2015 by Genetica DNA laboratories, Burlington, NC. HCT116 or BJ-hTERT cells were treated with TSA (100 ng/ml; Calbiochem) or nicotinamide (10mM; Sigma) for various time periods and whole cell extracts were prepared for Western blot analysis. Classical histone deacetylase HDAC1-HDAC6 expression plasmids and SIRT1 expression plasmid described earlier [5, 14, 75] were transfected in HK293T cells. HDAC1 level was downregulated by transfecting cells with HDAC1 siRNA (Dharmacon). Mutation of residues Lys 6, 7, 27, 31, 32 to Arg (K5R) in APE1-FLAG-tagged pCMV5.1 plasmid were generated using a site-directed mutagenesis Kit (Stratagene) following manufacturer's protocol. Exponentially growing HCT116 cells stably expressing APE1-shRNA were transfected with FLAGtagged PCMV5.1 expression plasmid containing wild

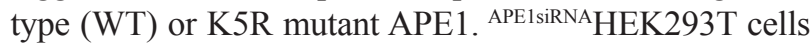
were treated with doxycycline (Dox; $1 \mathrm{ug} / \mathrm{ml}$ ) for 5 days to knockdown endogenous APE1 followed by transfection with FLAG-tagged WT APE1 or K5R mutant APE1 as described elsewhere [5, 9]. Cells were transfected using Lipofectamine 2000 following manufacturer's protocol and harvested after $48 \mathrm{~h}$ for cell lysate preparation. For Western blot analysis, $\alpha$-APE1[76], $\alpha$-AcAPE1, $\alpha$-HDAC1 (EMD Millipore; \# 06-720) Abs were used.

\section{In vitro acetylation/deacetylation assay}

Recombinant APE1 was purified as described previously [41], and was incubated with p300 HAT domain \pm $1 \mathrm{mM} \mathrm{AcCoA}$ for $2 \mathrm{~h}$ at $30^{\circ} \mathrm{C}$ and then $50 \mathrm{ng}$ of unmodified or AcAPE1 was used for Western blot analysis with Abs specific for AcAPE1 or APE1 [5, 9]. For in vitro deacetylation assay, lysates from HEK293T cells transfected with individual FLAG-tagged HDACs 1-6, were immunoprecipitated with $\alpha$-FLAG Ab cross-linked to agarose beads (Sigma; A2220) and eluted with FLAG peptide. $200 \mathrm{ng}$ of AcAPE1 was incubated with immunoaffinity purified $\mathrm{HDACs}$ at $37^{\circ} \mathrm{C}$ for $1 \mathrm{~h}$ and subjected to Western blot analysis.

\section{Apurinic/apyrimidinic (AP) endonuclease activity measurement assay}

A 43-mer oligonucleotide containing AP site analog tetrahydrofuran (THF) at nucleotide 31 (Midland Corp) prepared as described previously was 5 '-end-labeled with $\left[\gamma{ }^{32} \mathrm{P}\right]$ ATP using T4 polynucleotide kinase $[5,41,76]$. Following annealing to the complementary strand with A opposite THF, the duplex oligomer was purified by gel filtration column (Chroma Spin TE 10; Clontech). This THF-containing duplex oligomer was incubated with recombinant WT APE1, recombinant AcAPE1, mutant APE1 proteins, or cell lysates as described earlier at 37 ${ }^{\circ} \mathrm{C}$ for 3 min during which the reaction rate was linear in a $15 \mu \mathrm{l}$ reaction mixture containing $50 \mathrm{mM}$ Tris- $\mathrm{HCl} \mathrm{pH}$ 8.5, $50 \mathrm{mM} \mathrm{KCl}, 1 \mathrm{mM}$ DTT, $0.1 \mathrm{mM}$ EDTA and $100 \mu \mathrm{g} /$ $\mathrm{mL}$ bovine serum albumin and $2 \mathrm{mM} \mathrm{MgCl}$. The reaction was stopped with $10 \mu \mathrm{l} 80 \%$ formamide/40 $\mathrm{mM} \mathrm{NaOH}$ containing $0.05 \%$ xylene cyanol, followed by heating at $95^{\circ} \mathrm{C}$ for $5 \mathrm{~min}$; the samples were kept on ice until ran in denaturing gel electrophoresis in $20 \%$ polyacrylamide containing $8 \mathrm{M}$ urea to separate the substrate oligomer from the cleaved product. The gels were dried and the radioactivity was quantitated by phosphoimager analysis in a Storm system (Molecular Dynamics).

\section{AP site measurement assay}

Endogenous APE1 was downregulated in APE1siRNAHEK293T cells with Dox (Sigma; $1 \mu \mathrm{g} / \mathrm{ml}$ ) treatment [9]. Then the cells were transfected with WT, or mutant APE1 expression plasmids, as described above. Forty eight hours post transfection, total genomic DNA was isolated by Qiagen Dneasy kit following manufacturer's protocol. AP sites were measured using aldehyde reactive probe (Dodinjo, Japan) according to manufacturer's protocol.

\section{Comet assay}

Trevigen's FPG FLARE (Fragment Length Analysis using Repair Enzymes) Comet assay kit was used following manufacturer's protocol. Briefly, control and GOx treated cells were lysed in Comet slides in low melting agarose followed by incubation with FPG before alkaline electrophoresis. DNA in the nucleoid was visualized by SYBR Gold staining in a fluorescence microscope (EVOS FL auto, Life Technologies). Data analysis was performed using Open Comet of Image J program (NIH) in 50-100 randomly selected cells and plotted as mean Tail moment.

\section{Clonogenic assay}

HCT116 cells stably expressing APE1 shRNA were transfected with WT or mutant APE1 expression plasmid. After forty 48 hours of transfection, approximately 500 cells plated on $60-\mathrm{mm}$ dishes were treated with various doses of methylmethane sulphonate (MMS; Sigma; 0.5, 1, 1.5 and $2 \mathrm{mM}$ ) for 1 hour, washed in PBS and allowed to grow in fresh medium for two weeks until visible colonies 
appear. HCT116 cells expressing control shRNA used as a control. The colonies were fixed with $100 \%$ methanol, stained with Giemsa staining solution (1:50) and counted.

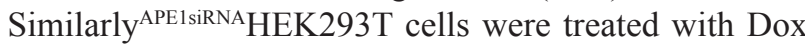
for 5-6 days to knockdown endogenous APE1. Cells were transfected with WT or mutant APE1 expression plasmid. Colony formation assay was performed after treatment GOx as described above.

\section{Statistical analysis}

Statistical analysis was done using Student T Test and $\mathrm{p}$ value less than 0.05 was considered significant.

\section{ACKNOWLEDGMENTS}

This research was funded by National Institute of Health (NIH) grant R01 CA148941 (to KKB), and a training fellowship (to SS) from the Keck Center for Quantitative Biomedical Sciences of the Gulf Coast Consortia, on the Computational Cancer Biology Training Program from the Cancer Prevention \& Research Institute of Texas (CPRIT No. RP101489). The authors kindly acknowledge Dr. Sankar Mitra (UTMB), for his help and support.

\section{CONFLICTS OF INTEREST}

The authors disclose no potential conflicts of interest.

\section{REFERENCES}

1. Li M and Wilson DM, 3rd. Human apurinic/apyrimidinic endonuclease 1. Antioxidants \& redox signaling. 2014; 20:678-707.

2. Demple B and Sung JS. Molecular and biological roles of Ape1 protein in mammalian base excision repair. DNA repair. 2005; 4:1442-1449.

3. Mitra S, Izumi T, Boldogh I, Bhakat KK, Hill JW and Hazra TK. Choreography of oxidative damage repair in mammalian genomes. Free radical biology \& medicine. 2002; 33:15-28.

4. Doetsch PW and Cunningham RP. The enzymology of apurinic/apyrimidinic endonucleases. Mutation research. 1990; 236:173-201.

5. Chattopadhyay R, Das S, Maiti AK, Boldogh I, Xie J, Hazra TK, Kohno K, Mitra S and Bhakat KK. Regulatory role of human AP-endonuclease (APE1/Ref-1) in YB-1-mediated activation of the multidrug resistance gene MDR1. Molecular and cellular biology. 2008; 28:7066-7080.

6. Fantini D, Vascotto C, Deganuto M, Bivi N, Gustincich S, Marcon G, Quadrifoglio F, Damante G, Bhakat KK, Mitra $\mathrm{S}$ and Tell G. APE1/Ref-1 regulates PTEN expression mediated by Egr-1. Free Radic Res. 2008; 42:20-29.
7. Ray S, Lee C, Hou T, Bhakat KK and Brasier AR. Regulation of signal transducer and activator of transcription 3 enhanceosome formation by apurinic/ apyrimidinic endonuclease 1 in hepatic acute phase response. Mol Endocrinol. 2010; 24:391-401.

8. Sengupta S, Chattopadhyay R, Mantha AK, Mitra S and Bhakat KK. Regulation of mouse-renin gene by apurinic/ apyrimidinic-endonuclease 1 (APE1/Ref-1) via recruitment of histone deacetylase 1 corepressor complex. Journal of hypertension. 2012; 30:917-925.

9. Sengupta S, Mantha AK, Mitra S and Bhakat KK. Human AP endonuclease (APE1/Ref-1) and its acetylation regulate YB-1-p300 recruitment and RNA polymerase II loading in the drug-induced activation of multidrug resistance gene MDR1. Oncogene. 2011; 30:482-493.

10. Sengupta S, Mitra S and Bhakat KK. Dual regulatory roles of human AP-endonuclease (APE1/Ref-1) in CDKN1A/p21 expression. PloS one. 2013; 8:e68467.

11. Bhakat KK, Mantha AK and Mitra S. Transcriptional regulatory functions of mammalian AP-endonuclease (APE1/Ref-1), an essential multifunctional protein. Antioxidants \& redox signaling. 2009; 11:621-638.

12. Georgiadis MM, Luo M, Gaur RK, Delaplane S, Li X and Kelley MR. Evolution of the redox function in mammalian apurinic/apyrimidinic endonuclease. Mutation research. 2008; 643:54-63.

13. Evans AR, Limp-Foster M and Kelley MR. Going APE over ref-1. Mutation research. 2000; 461:83-108.

14. Bhakat KK, Izumi T, Yang SH, Hazra TK and Mitra S. Role of acetylated human AP-endonuclease (APE1/Ref-1) in regulation of the parathyroid hormone gene. Embo J. 2003; 22:6299-6309.

15. Bhattacharyya A, Chattopadhyay R, Burnette BR, Cross JV, Mitra S, Ernst PB, Bhakat KK and Crowe SE. Acetylation of apurinic/apyrimidinic endonuclease-1 regulates Helicobacter pylori-mediated gastric epithelial cell apoptosis. Gastroenterology. 2009; 136:2258-2269.

16. Lirussi L, Antoniali G, Vascotto C, D'Ambrosio C, Poletto M, Romanello M, Marasco D, Leone M, Quadrifoglio F, Bhakat KK, Scaloni A and Tell G. Nucleolar accumulation of APE1 depends on charged lysine residues that undergo acetylation upon genotoxic stress and modulate its BER activity in cells. Molecular biology of the cell. 2012; 23:4079-4096.

17. Busso CS, Iwakuma $\mathrm{T}$ and Izumi T. Ubiquitination of mammalian AP endonuclease (APE1) regulated by the $\mathrm{p} 53$ MDM2 signaling pathway. Oncogene. 2009; 28:1616-1625.

18. Busso CS, Lake MW and Izumi T. Posttranslational modification of mammalian AP endonuclease (APE1). Cellular and molecular life sciences : CMLS. 2010; 67:3609-3620.

19. Busso CS, Wedgeworth $\mathrm{CM}$ and Izumi T. Ubiquitination of human AP-endonuclease 1 (APE1) enhanced by T233E substitution and by CDK5. Nucleic acids research. 2011; 39:8017-8028. 
20. Qu J, Liu GH, Huang B and Chen C. Nitric oxide controls nuclear export of APE1/Ref-1 through S-nitrosation of cysteines 93 and 310. Nucleic acids research. 2007; 35:2522-2532.

21. Tang $\mathrm{CH}$, Wei $\mathrm{W}$ and Liu L. Regulation of DNA repair by S-nitrosylation. Biochimica et biophysica acta. 2012; 1820:730-735.

22. Huang E, Qu D, Zhang Y, Venderova K, Haque ME, Rousseaux MW, Slack RS, Woulfe JM and Park DS. The role of Cdk5-mediated apurinic/apyrimidinic endonuclease 1 phosphorylation in neuronal death. Nature cell biology. 2010; 12:563-571.

23. Antoniali G, Lirussi L, Poletto M and Tell G. Emerging roles of the nucleolus in regulating the DNA damage response: the noncanonical DNA repair enzyme APE1/ Ref-1 as a paradigmatical example. Antioxidants \& redox signaling. 2014; 20:621-639.

24. Vascotto C, Lirussi L, Poletto M, Tiribelli M, Damiani D, Fabbro D, Damante G, Demple B, Colombo E and Tell G. Functional regulation of the apurinic/apyrimidinic endonuclease 1 by nucleophosmin: impact on tumor biology. Oncogene. 2014; 33:2876-2887.

25. Izumi T, Brown DB, Naidu CV, Bhakat KK, Macinnes MA, Saito H, Chen DJ and Mitra S. Two essential but distinct functions of the mammalian abasic endonuclease. Proceedings of the National Academy of Sciences of the United States of America. 2005; 102:5739-5743.

26. Bhakat KK, Hazra TK and Mitra S. Acetylation of the human DNA glycosylase NEIL2 and inhibition of its activity. Nucleic acids research. 2004; 32:3033-3039.

27. Bhakat KK, Mokkapati SK, Boldogh I, Hazra TK and Mitra S. Acetylation of human 8-oxoguanine-DNA glycosylase by p300 and its role in 8-oxoguanine repair in vivo. Molecular and cellular biology. 2006; 26:1654-1665.

28. Abbotts R and Madhusudan S. Human AP endonuclease 1 (APE1): from mechanistic insights to druggable target in cancer. Cancer treatment reviews. 2010; 36:425-435.

29. Bapat A, Fishel ML and Kelley MR. Going ape as an approach to cancer therapeutics. Antioxidants \& redox signaling. 2009; 11:651-668.

30. Bapat A, Glass LS, Luo M, Fishel ML, Long EC, Georgiadis MM and Kelley MR. Novel small-molecule inhibitor of apurinic/apyrimidinic endonuclease 1 blocks proliferation and reduces viability of glioblastoma cells. J Pharmacol Exp Ther. 2010; 334:988-998.

31. Bobola MS, Blank A, Berger MS, Stevens BA and Silber JR. Apurinic/apyrimidinic endonuclease activity is elevated in human adult gliomas. Clin Cancer Res. 2001; 7:3510-3518.

32. Bobola MS, Finn LS, Ellenbogen RG, Geyer JR, Berger MS, Braga JM, Meade EH, Gross ME and Silber JR. Apurinic/apyrimidinic endonuclease activity is associated with response to radiation and chemotherapy in medulloblastoma and primitive neuroectodermal tumors. Clin Cancer Res. 2005; 11:7405-7414.
33. Fishel ML, He Y, Reed AM, Chin-Sinex H, Hutchins GD, Mendonca MS and Kelley MR. Knockdown of the DNA repair and redox signaling protein Ape1/Ref-1 blocks ovarian cancer cell and tumor growth. DNA repair. 2008; 7:177-186.

34. Fishel ML and Kelley MR. The DNA base excision repair protein Ape1/Ref-1 as a therapeutic and chemopreventive target. Mol Aspects Med. 2007; 28:375-395.

35. Xie J, Zhang L, Li M, Du J, Zhou L, Yang S, Zeng L, Li Z, Wang $\mathrm{G}$ and Wang D. Functional analysis of the involvement of apurinic/apyrimidinic endonuclease 1 in the resistance to melphalan in multiple myeloma. BMC cancer. 2014; 14:11.

36. Yoo DG, Song YJ, Cho EJ, Lee SK, Park JB, Yu JH, Lim SP, Kim JM and Jeon BH. Alteration of APE1/ref-1 expression in non-small cell lung cancer: the implications of impaired extracellular superoxide dismutase and catalase antioxidant systems. Lung Cancer. 2008; 60:277-284.

37. Bhakat KK, Sengupta S, Adeniyi VF, Roychoudhury S, Nath S, Bellot LJ, Feng D, Mantha AK, Sinha M, Qiu S and Luxon BA. Regulation of limited N-terminal proteolysis of APE1 in tumor via acetylation and its role in cell proliferation. Oncotarget. 2016, 7 :22590-22604. doi: 10.18632/oncotarget.8026.

38. Yoshida M, Kijima M, Akita M and Beppu T. Potent and specific inhibition of mammalian histone deacetylase both in vivo and in vitro by trichostatin A. The Journal of biological chemistry. 1990; 265:17174-17179.

39. Yamamori T, DeRicco J, Naqvi A, Hoffman TA, Mattagajasingh I, Kasuno K, Jung SB, Kim CS and Irani K. SIRT1 deacetylates APE1 and regulates cellular base excision repair. Nucleic acids research. 2010; 38:832-845.

40. Borra MT, Smith BC and Denu JM. Mechanism of human SIRT1 activation by resveratrol. The Journal of biological chemistry. 2005; 280:17187-17195.

41. Mantha AK, Oezguen N, Bhakat KK, Izumi T, Braun W and Mitra S. Unusual role of a cysteine residue in substrate binding and activity of human AP-endonuclease 1. Journal of molecular biology. 2008; 379:28-37.

42. McNeill DR, Lam W, DeWeese TL, Cheng YC and Wilson DM, 3rd. Impairment of APE1 function enhances cellular sensitivity to clinically relevant alkylators and antimetabolites. Molecular cancer research : MCR. 2009; 7:897-906.

43. McNeill DR and Wilson DM, 3rd. A dominant-negative form of the major human abasic endonuclease enhances cellular sensitivity to laboratory and clinical DNAdamaging agents. Molecular cancer research : MCR. 2007; 5:61-70.

44. Simeonov A, Kulkarni A, Dorjsuren D, Jadhav A, Shen M, McNeill DR, Austin CP and Wilson DM, 3rd. Identification and characterization of inhibitors of human apurinic/ apyrimidinic endonuclease APE1. PloS one. 2009; 4:e5740.

45. Atamna H, Cheung I and Ames BN. A method for detecting abasic sites in living cells: age-dependent changes in base excision repair. Proceedings of the National Academy of Sciences of the United States of America. 2000; 97:686-691. 
46. Salazar JJ and Van Houten B. Preferential mitochondrial DNA injury caused by glucose oxidase as a steady generator of hydrogen peroxide in human fibroblasts. Mutation research. 1997; 385:139-149.

47. Izumi $\mathrm{T}$ and Mitra $\mathrm{S}$. Deletion analysis of human AP-endonuclease: minimum sequence required for the endonuclease activity. Carcinogenesis. 1998; 19:525-527.

48. Demple B and Harrison L. Repair of oxidative damage to DNA: enzymology and biology. Annu Rev Biochem. 1994; 63:915-948.

49. Lindahl $\mathrm{T}$ and Andersson A. Rate of chain breakage at apurinic sites in double-stranded deoxyribonucleic acid. Biochemistry. 1972; 11:3618-3623.

50. Tornaletti S, Maeda LS and Hanawalt PC. Transcription arrest at an abasic site in the transcribed strand of template DNA. Chemical research in toxicology. 2006; 19:1215-1220.

51. Fung $\mathrm{H}$ and Demple B. Distinct roles of Ape1 protein in the repair of DNA damage induced by ionizing radiation or bleomycin. The Journal of biological chemistry. 2011; 286:4968-4977.

52. Boiteux S and Guillet M. Abasic sites in DNA: repair and biological consequences in Saccharomyces cerevisiae. DNA repair. 2004; 3:1-12.

53. Viswesh V, Gates K and Sun D. Characterization of DNA damage induced by a natural product antitumor antibiotic leinamycin in human cancer cells. Chemical research in toxicology. 2010; 23:99-107.

54. Loeb LA and Preston BD. Mutagenesis by apurinic/ apyrimidinic sites. Annual review of genetics. 1986; 20:201-230

55. Reuter S, Gupta SC, Chaturvedi MM and Aggarwal BB. Oxidative stress, inflammation, and cancer: how are they linked? Free radical biology \& medicine. 2010; 49:1603-1616.

56. Toyokuni S, Okamoto K, Yodoi J and Hiai H. Persistent oxidative stress in cancer. FEBS letters. 1995; 358:1-3.

57. Chastain PD, 2nd, Nakamura J, Rao S, Chu H, Ibrahim JG, Swenberg JA and Kaufman DG. Abasic sites preferentially form at regions undergoing DNA replication. FASEB journal. 2010; 24:3674-3680.

58. Chastain PD, 2nd, Nakamura J, Swenberg J and Kaufman D. Nonrandom AP site distribution in highly proliferative cells. FASEB journal. 2006; 20:2612-2614.

59. Nowsheen S, Wukovich RL, Aziz K, Kalogerinis PT, Richardson CC, Panayiotidis MI, Bonner WM, Sedelnikova $\mathrm{OA}$ and Georgakilas AG. Accumulation of oxidatively induced clustered DNA lesions in human tumor tissues. Mutation research. 2009; 674:131-136.

60. Sutherland BM, Bennett PV, Cintron NS, Guida P and Laval J. Low levels of endogenous oxidative damage cluster levels in unirradiated viral and human DNAs. Free radical biology \& medicine. 2003; 35:495-503.
61. Masuda Y, Bennett RA and Demple B. Dynamics of the interaction of human apurinic endonuclease (Ape1) with its substrate and product. The Journal of biological chemistry. 1998; 273:30352-30359.

62. Masuda Y, Bennett RA and Demple B. Rapid dissociation of human apurinic endonuclease (Ape1) from incised DNA induced by magnesium. The Journal of biological chemistry. 1998; 273:30360-30365.

63. Mol CD, Izumi T, Mitra S and Tainer JA. DNA-bound structures and mutants reveal abasic DNA binding by APE1 and DNA repair coordination [corrected]. Nature. 2000; 403:451-456.

64. Yacoub A, Kelley MR and Deutsch WA. The DNA repair activity of human redox/repair protein APE/Ref-1 is inactivated by phosphorylation. Cancer research. 1997; 57:5457-5459.

65. Hsieh MM, Hegde V, Kelley MR and Deutsch WA. Activation of APE/Ref-1 redox activity is mediated by reactive oxygen species and PKC phosphorylation. Nucleic acids research. 2001; 29:3116-3122.

66. Fritz $\mathrm{G}$ and Kaina B. Phosphorylation of the DNA repair protein APE/REF-1 by CKII affects redox regulation of AP-1. Oncogene. 1999; 18:1033-1040.

67. Chan HM and La Thangue NB. p300/CBP proteins: HATs for transcriptional bridges and scaffolds. Journal of cell science. 2001; 114:2363-2373.

68. Liu JL, Wang XY, Huang BX, Zhu F, Zhang RG and Wu G. Expression of CDK5/p35 in resected patients with non-small cell lung cancer: relation to prognosis. Medical oncology (Northwood, London, England). 2011; 28:673-678.

69. Brooks $\mathrm{CL}$ and $\mathrm{Gu}$ W. Ubiquitination, phosphorylation and acetylation: the molecular basis for $\mathrm{p} 53$ regulation. Current opinion in cell biology. 2003; 15:164-171.

70. Al-Safi RI, Odde S, Shabaik Y and Neamati N. Smallmolecule inhibitors of APE1 DNA repair function: an overview. Current molecular pharmacology. 2012; 5:14-35.

71. Kievit FM, Stephen ZR, Wang K, Dayringer CJ, Sham JG, Ellenbogen RG, Silber JR and Zhang M. Nanoparticle mediated silencing of DNA repair sensitizes pediatric brain tumor cells to gamma-irradiation. Molecular oncology. 2015; 9:1071-1080.

72. Qian C, Li M, Sui J, Ren T, Li Z, Zhang L, Zhou L, Cheng $Y$ and Wang D. Identification of a novel potential antitumor activity of gossypol as an APE1/Ref-1 inhibitor. Drug design, development and therapy. 2014; 8:485-496.

73. Rai G, Vyjayanti VN, Dorjsuren D, Simeonov A, Jadhav A, Wilson DM and Maloney DJ. (2010). Small Molecule Inhibitors of the Human Apurinic/apyrimidinic Endonuclease 1 (APE1). Probe Reports from the NIH Molecular Libraries Program. (Bethesda (MD): National Center for Biotechnology Information (US)).

74. Srinivasan A, Wang L, Cline CJ, Xie Z, Sobol RW, Xie $\mathrm{XQ}$ and Gold B. Identification and characterization of 
human apurinic/apyrimidinic endonuclease-1 inhibitors. Biochemistry. 2012; 51:6246-6259.

75. Wang F, Chan CH, Chen K, Guan X, Lin HK and Tong Q. Deacetylation of FOXO3 by SIRT1 or SIRT2 leads to Skp2-mediated FOXO3 ubiquitination and degradation. Oncogene. 2012; 31:1546-1557.
76. Ramana CV, Boldogh I, Izumi T and Mitra S. Activation of apurinic/apyrimidinic endonuclease in human cells by reactive oxygen species and its correlation with their adaptive response to genotoxicity of free radicals. Proceedings of the National Academy of Sciences of the United States of America. 1998; 95:5061-5066. 